\title{
Imaging of Cholangiocarcinoma
}

\author{
Susann-Cathrin Olthof Ahmed Othman Stephan Clasen Christina Schraml \\ Konstantin Nikolaou Malte Bongers
}

Department of Diagnostic and Interventional Radiology, University Hospital of Tübingen, Tübingen, Germany

\section{Keywords}

Cross-sectional imaging · Cholangiocarcinoma .

Computed tomography · Magnetic resonance imaging ·

Positron emission tomography

\section{Summary}

Cholangiocarcinoma (CC) is the second most common primary hepatobiliary tumour, and it is increasing in incidence. Imaging characteristics, behaviour, and therapeutic strategies in CC differ significantly, depending on the morphology and location of the tumour. In cross-sectional imaging, CCs can be classified according to the growth pattern (mass-forming, periductal infiltrating, intraductal) and the location (intrahepatic, perihilar, extrahepatic/distal). The prognosis of CC is unfavourable and surgical resection is the only curative treatment option; thus, early diagnosis (also in recurrent disease) and accurate staging including the evaluation of lymph node involvement and vascular infiltration is crucial. However, the diagnostic evaluation of $\mathrm{CC}$ is challenging due to the heterogeneous nature of the tumour. Diagnostic modalities used in the imaging of $\mathrm{CC}$ include transabdominal ultrasound, endosonography, computed tomography, magnetic resonance imaging with cholangiopancreatography, and hybrid imaging such as positron emission tomography/computed tomography. In this review, the potential of cross-sectional imaging modalities in primary staging, treatment monitoring, and detection of recurrent disease will be discussed.

(C) 2016 S. Karger GmbH, Freiburg

\section{Introduction}

Cholangiocarcinoma (CC) is the most frequent malignant tumour of the biliary tract, accounting for $10-20 \%$ of all primary liver tumours $[1,2]$. The vast majority $(95 \%)$ of CC are adenocarcinomas with a high proportion of fibrous stroma $[3,4]$. The prevalence of CC shows geographic variations, with the highest prevalence being found in Southeast Asia [2]. In the US, the incidence of CC has been steadily increasing over the past decades, varying from 0.72 to 0.88 per 100,000 [5]. Despite recent advances in patient care, surgical resection of the tumour remains the only potentially curable therapy, leading to a 5-year survival of $30-35 \%$ [6]. CC may occur at any segment of the bile duct, i.e. from the terminal ductules to the ampulla of Vater. Therefore, intrahepatic (iCC) and extrahepatic (eCC) tumour localizations are differentiated, and the iCC is subdivided into peripheral and perihilar CC (pCC), the latter also being called Klatskin tumour. In detail, $6-8 \%$ of CC are localized peripherally, $50-67 \%$ perihilar, and $27-42 \%$ occur extrahepatically [7]. Up to now, the aetiology of CC is not fully understood, but several risk factors like primary sclerosing cholangitis (PSC), liver fluke infestations (Opisthorchis viverrini, Clonorchis sinensis), hepatolithiasis, Thorotrast exposure, and choledochal cysts have been identified [8]. In accordance with the morphologic classification system proposed by the Liver Cancer Study Group of Japan [9], iCC can be classified into three types based on the dominating morphologic feature: mass-forming (the most common), periductal infiltrating, and intraductal growth (fig. 1). Additionally, the growth pattern of eCC is commonly classified as nodular, sclerosing, or papillary [10]. Imaging modalities like ultrasound (US), computed tomography (CT), magnetic resonance imaging (MRI), and also positron emission tomography/computer tomography (PET/CT) are of particular importance in the diagnosis, primary staging, and restaging of CC.

\section{Ultrasound}

When clinical and laboratory findings are suggestive of CC, transabdominal US is commonly used in the initial phase of CC diagnostics. The technique is helpful to investigate the cause of bile duct obstruction and to characterize liver lesions. Recent technical

\section{KARGER}

(๑) 2016 S. Karger GmbH, Freiburg

Fax +497614520714
Dr. med. Malte Bongers

Abteilung für Diagnostische und Interventionelle Radiologie Universitätsklinikum Tübingen

Hoppe-Seyler-Straße 3, 72076 Tübingen, Germany malte.bongers@med.uni-tuebingen.de 


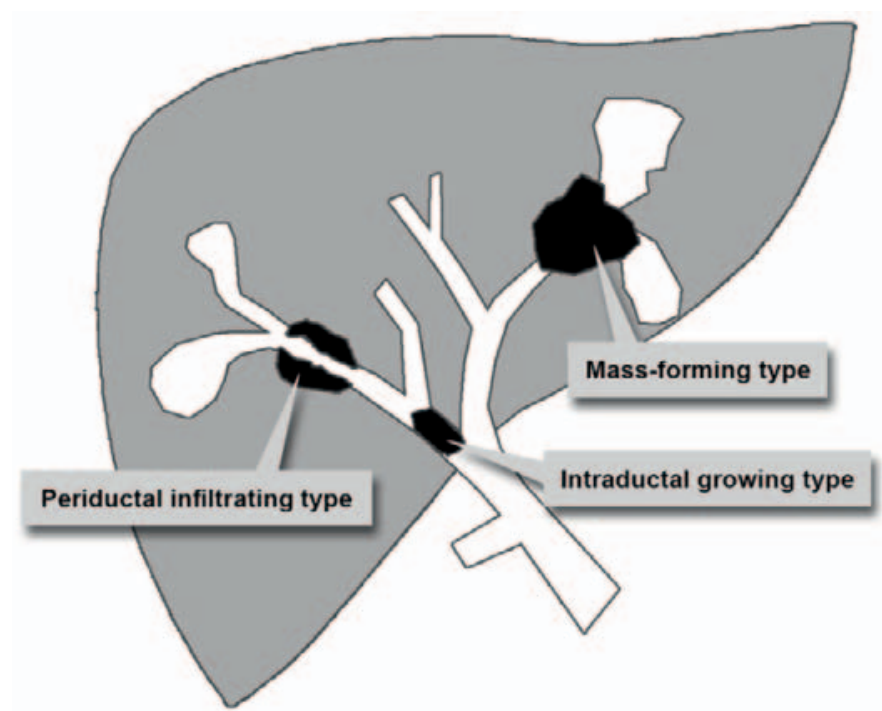

Fig. 1. Schematic illustration of the three most common morphologic features of iCC according to the Liver Cancer Study Group of Japan [13].

advances, including transabdominal and endoscopic US applications, and also the use of contrast-enhanced US indicate great potential for the evaluation of both luminal and extraluminal masses in CC diagnosis. A detailed discussion of the role of US in the diagnosis of CC, however, is beyond the scope of this review as it is mainly focusing on the cross-sectional imaging techniques CT, MRI, and PET/CT. When CC is suspected in US due to dilatation of the intrahepatic biliary or an intrahepatic mass, further investigations are generally performed with a second-level imaging modality such as CT or MRI.

\section{Computed Tomography}

CT is performed in up to $90 \%$ of suspected CC, offering the opportunity of assessing the full extension of the tumour and determining potential surgical resectability. Additionally, pathological details, such as vascular infiltration and the presence of lymph node metastasis, can be estimated. In primary staging of CC, multiphase CT scanning protocols are recommended. By means of precontrast scanning (fig. 2A), intraductal stones (as one of the risk factors of CC) can be identified [11, 12]. In the arterial phase, the exact arterial anatomy of the liver and surrounding structures can be displayed, allowing for planning the surgical method in detail (fig. 2B). In the portal venous phase (fig. 2C), CC mainly show incomplete rim-like contrast enhancement at the tumour periphery and low intratumoural attenuation, increasing the precision in estimating the tumour size and the detection of satellite nodules [13] In late-phase scans (5-10 min after contrast injection), a late enhancement of the tumour can be observed, representing the amount of fibrous stroma. In case of tumour necrosis and/or mucin-containing cells, the delayed enhancement may disappear [14]. Due to the volumetric scanning technique, CT images can be used in preoperative management to estimate the liver volume and potential liver remnants, avoiding postoperative small-for-size-syndrome $[15,16]$. Based on this information, alternative surgical procedures like 'associating liver partition and portal vein ligation for staged hepatectomy' (ALPPS) can be considered [17]. As already described, the main morphologic pattern of iCC can be characterized as mass-forming, periductal infiltrating, and intraductal growth which can be visualized using CT imaging.

Mass-forming iCC usually appears as a homogeneous low-attenuating mass on non-contrast-enhanced scans. After contrast media injection, an irregular peripheral enhancement with only minor enhancement in the centre of the tumour can be observed [13]. The degree of central contrast enhancement is increasing from arterial- to late-phase images and depends on the amount of central tumour fibrosis. Additional findings like capsular retraction and dilatation of bile ducts distal to the mass are typical. Narrowing of portal or hepatic veins is observed less frequently. Vascular invasion typically leads to lobar or segmental atrophy of the liver [18]. Periductal infiltrating iCC is characterized by a growth pattern along the bile duct without mass formation. The involved bile ducts can be dilated or narrowed but show diffuse periductal thickening with increased enhancement from arterial- to delayedphase CT. Usually, a dilatation of the distal bile ducts is present [13]. Intraductal growth of iCC is primarily characterized by an irregular duct calibre. Usually, focal duct ectasia with or without a visible intraductal mass can be detected. In case of intraductal mass, a hypoattenuation with irregular margins can be observed on pre-contrast imaging, showing increasing contrast enhancement in portal venous- and delayed-phase images [13]. Imaging-based preoperative differentiation of growth pattern of eCC into nodular, sclerosing, and papillary remains challenging and does not seem to be of primary importance concerning the surgical strategy [19].

\section{Primary Staging of Intrahepatic Cholangiocarcinoma}

The literature on staging iCC by CT is poor. Prognosis of iCC depends on the tumour size, lymph node metastasis, quantity of hepatic tumours or intrahepatic metastasis, and vascular invasion. In comparison with MRI, CT seems to be more sensitive in detecting vascular involvement and extrahepatic invasion; therefore, it may be advantageous in the primary staging of iCC [20]. Additionally, CT might be more accurate than MRI in predicting the resectability of iCC, with an accuracy of up to $88 \%$ and a negative predictive value of $85-100 \%$ [21].

\section{Primary Staging of Perihilar Cholangiocarcinoma}

Based on 16 articles, a meta-analysis from 2012 estimated the accuracy of CT in staging pCC [22]. When judging the ductal extent of the tumour, CT showed an overall diagnostic accuracy of $86 \%$ (95\% confidence interval (CI): 77-92\%). Portal vein involvement was detected by CT with a sensitivity of $89 \%$ (95\% CI: $80-$ $94 \%$ ) and a specificity of $92 \%$ (95\% CI: $85-96 \%$ ). CT diagnosis of hepatic artery involvement showed a sensitivity of $84 \%$ (95\% CI: $63-94 \%)$ and a specificity of $93 \%$ (95\% CI: 69-99\%). The accuracy of CT in detecting lymph node metastasis seems to be limited, with 
Fig. 2. CT images of an iCC. A In non-contrast enhanced images, a hypodense area is present in liver segment V-VI/VII-VIII. B Tumour vascularity can be visualized in major intensity projections of arterial-phase images. C The exact tumour size with pe-
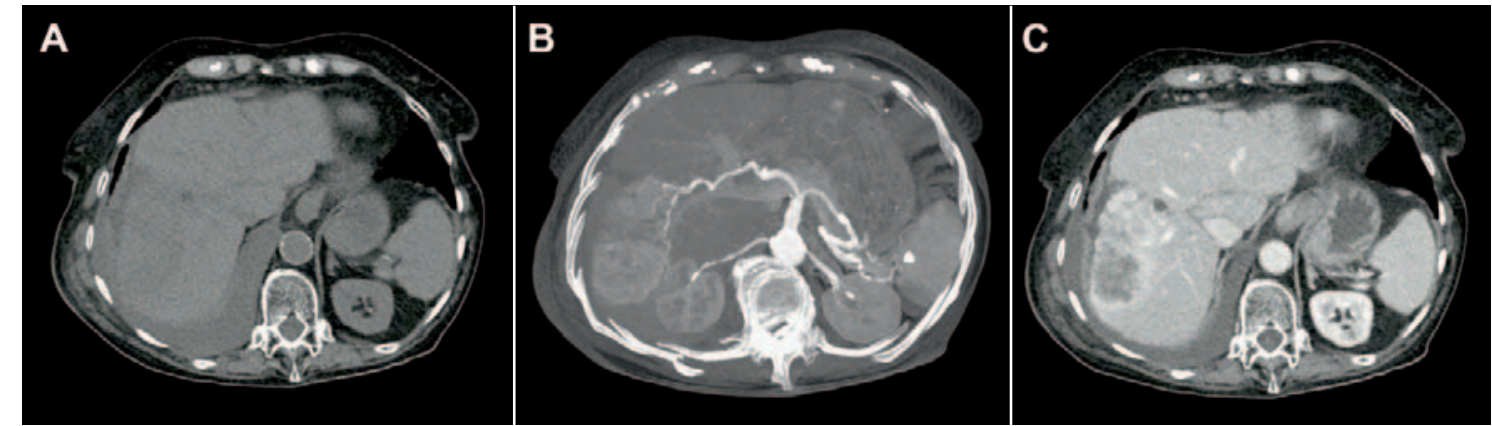

ripheral enhancement and typical hypodense areas in the lesion centre are shown in portal-venous phase CT images.

a reported sensitivity of $61 \%$ (95\% CI: $28-86 \%)$ and a specificity of $88 \%$ (95\% CI: 74-95\%). Concerning the evaluation of distant metastasis, CT showed a sensitivity of $67 \%$ and a specificity of $94 \%$. So far, there are only a few studies which directly compare the accuracy of CT and MRI in the staging of PCC with controversial findings. In one study, the accuracy of estimating the intraductal tumour size was a little higher for MRI (71\%) than for CT (64\%) [23]. A second study favoured CT with an accuracy of $80 \%$ (MRI 75\%) for the estimation of the intraductal tumour size [24].

\section{Primary Staging of Extrahepatic Cholangiocarcinoma}

In the staging of eCC, determining the exact tumour localization and its longitudinal and lateral expansion is of primary importance as the surgical resection method and the patient's prognosis both depend on these factors. Tumour localization is commonly described by using the modified Bismuth-Corlette classification (type I involves the common hepatic duct, type II involves the hepatic duct bifurcation, types IIIA and IIIB involve the right and left hepatic ducts, respectively, and type IV involves both the right and left hepatic ducts) [25]. Following the current $\mathrm{T}$ staging based on the seventh edition of the American Joint Committee on Cancer (AJCC) staging system [26], proximal and distal eCC are differentiated and $\mathrm{T} 1$ tumours are limited to the bile duct, whereas $\mathrm{T} 2$ tumours extend beyond the wall of the bile duct. With imaging (CT, MRI) and even in histopathology, the exact determination of the border of the bile duct is difficult [27]. Therefore, an accurate preoperative distinction of $\mathrm{T} 1$ and $\mathrm{T} 2$ tumours does not seem to be possible. Up to now, little is known about the exact accuracy of $\mathrm{CT}$ in the staging of eCC. The main issue constitutes the determination of tumour spread outside the wall of the bile duct, potentially affecting adjacent structures like the portal vein and hepatic artery, the hepatoduodenal ligament, and the head of the pancreas in T3 and T4 tumours. Preoperative CT-based three-dimensional space (3D) angiography and multiphase fusion imaging technique may be helpful in planning the surgical intervention, potentially minimizing operating time, the extent of surgical resection, and blood loss [28].

\section{Prognosis}

In mass-forming iCC, the degree of enhancement on delayedphase CT scans seems to be a potential prognostic indicator. Asay- ama et al. [29] postulated a worse prognosis for patients with an intratumoural enhancement greater than two thirds on the delayed images. Two studies including 42 and 70 patients with iCC and operative resection showed strong correlations between augmented arterial tumour enhancement in preoperative CT, histopathological vascularity, and higher survival rate. Histological examination of these tumours demonstrated a smaller amount of fibrotic tissue and necrosis but a higher cellularity [30,31]. The estimated overall accuracy of CT in evaluating tumour resectability ranges between 60 and $75 \%[32,33]$.

\section{Recurrent Disease}

The probability of local recurrence and distant metastasis in iCC is high. Hyder et al. [34] analysed recurrence patterns of 301 patients who underwent surgical resection of iCC between 1990 and 2011. During the follow-up of 31 months, 53.5\% patients developed recurrent, $60.9 \%$ intrahepatic, $21 \%$ extrahepatic, and $18.6 \%$ simultaneous intra- and extrahepatic disease [34]. Most frequently, metastases are found in the lungs (24\%), peritoneum (18\%), and bones (14\%) [35]. Therefore, follow-up restaging CT in CC patients has to cover the thorax and main part of the skeletal system.

\section{Novel Computed Tomography Techniques}

Contrast-enhanced dual-energy CT (DECT) offers the opportunity of calculating virtual non-enhanced images, thus allowing the detection of bile duct stones without pre-contrast scanning [36]. Additionally, hepatic calcifications can be accurately diagnosed when using this technique. Due to the utilization of iodine mapping and monoenergetic extrapolation, the detectability of vascularity of the liver and thrombosis can be improved [37, 38]. Marin et al. [39] showed a significant improvement of the conspicuity of hypervascular liver tumours by using low-keV (kilo electron volt) monoenergetic reconstructions. Analysing the virtual spectral curves from monoenergetic reconstructions of focal liver lesions might be helpful in differentiating benign from malign liver tumours [40]. Potential risk factors of CC like steatosis and iron overload can be estimated by employing dedicated DECT post-processing techniques $[41,42]$. Functional imaging of volume perfusion CT (VPCT) can help to assess the exact vascularization of the tumour in CC. Compared with the normal parenchyma, CC usually shows increased 

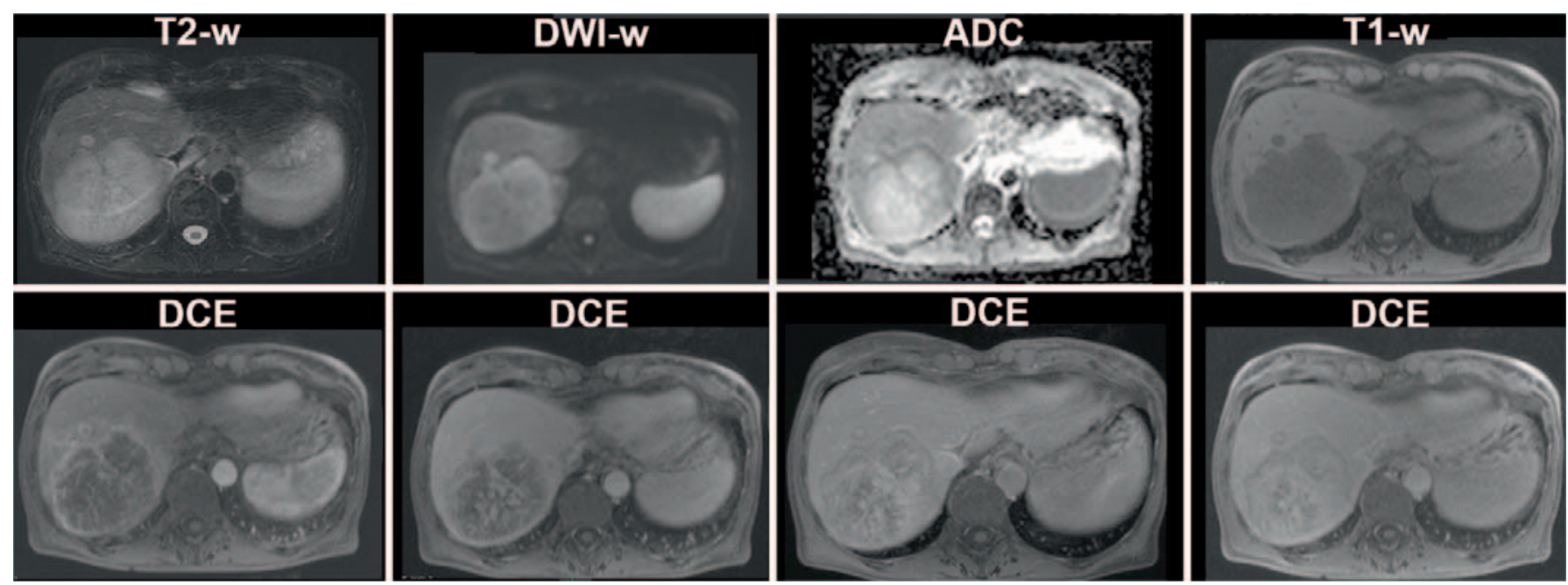

Fig. 3. MRI of a 64-year-old female patient with mass-forming CC of the right liver lobe. The CC appears hyperintense on $\mathrm{T} 2$-weighted images with diffusion restriction on DWI and shows a typical contrast enhancement pattern on the DCE-MRI.

hepatic arterial perfusion, increased blood volume, and decreased portal perfusion $[43,44]$. Due to novel treatment regimens such as anti-angiogenetic drugs or targeted therapies, VPCT might be a helpful monitoring tool for judging treatment response by revealing changes in tumour vascularization in the absence of significant changes in tumour size [18]. Novel techniques in CT post-processing like frequency-selective non-linear blending may offer additional benefits in the diagnosis of CC [45].

\section{Magnetic Resonance Imaging}

In recent years, the field of abdominal MRI experienced a tremendous development, resulting in more reliable imaging sequences and increased image quality, which in turn led to improved tumour detectability, for instance in the case of liver tumours [46]. In comparison to other imaging techniques, MRI enables higher tissue contrast. According to current guidelines, MRI is the modality of choice for the diagnosis and staging of CC [47].

For the assessment of CC, comprehensive imaging protocols not only including the liver but also covering the biliary tract and pancreas should be applied in order to rule out other malignancies such as pancreatic head adenocarcinoma. A typical MRI protocol for the assessment of CC encompasses magnetic resonance cholangiopancreatography (MRCP), conventional T1- and T2-weighted sequences, as well as diffusion-weighted imaging (DWI) and dynamic contrast-enhanced (DCE) MRI [48]. Furthermore, MRI with hepatocyte-specific contrast agents is often performed for the assessment of CC [49].

$\mathrm{MRCP}$ is a non-contrast MRI technique for assessing the biliary system [50]. In fact, MRCP is the most accurate non-invasive imaging technique for the assessment of the biliary system [51]. It allows accurate tumour assessment in both proximal and distal bile ducts [51]. For appropriate depiction of the biliary system in MRCP, heavily T2-weighted sequences with very long echo time are obtained, resulting in high (i.e. hyperintense) fluid signal in the bile ducts and low (i.e. suppressed) background signal [48]. MRCP sequences are usually acquired using a combination of thick-slab radial $\mathrm{T} 2$ sequences and thin 3D T2 sequences. The thick-slab sequences provide a good overview of the biliary system with a good suppression of the surrounding tissue, whereas the thin $3 \mathrm{D}$ T2 sequences provide high spatial resolution and allow for the detection of small abnormalities in the bile ducts such as small masses and/or strictures [48].

In addition to MRCP, which is useful for depicting intraductal CC tumour growth, DWI, DCE-MRI, and late-phase sequences with hepatocyte-specific contrast agents are helpful in the assessment of extraductal tumour growth as well as in the depiction of tumour masses within dilated bile ducts $[48,52]$.

MRI, especially in combination with MRCP, has a high diagnostic accuracy for the assessment of CC. Park et al. [53] showed that MRCP and MRI revealed a comparable diagnostic accuracy with invasive cholangiography combined with CT imaging for the detection of CC.

Preoperative MRI is commonly performed in patients with CC in order to assess the extent, resectability, and vascular involvement of the tumour. It is also employed for road mapping of the hepatic vessels and for visualization of vascular anatomic variants. Accurate preoperative assessment of liver vasculature has been shown to significantly affect the surgical outcome in patients with CC [54].

Besides the preoperative staging and follow-up imaging of CC, MRI and MRCP play a central role in the surveillance of patients with an increased risk of CC, e.g. those with PSC. The lifetime incidence of CC in patients with PSC ranges from 5 to 10\% [48]. According to recent recommendations, MRI including MRCP is appropriate for the surveillance of those patients [55]. 


\section{Magnetic Resonance Imaging of Mass-Forming}

\section{Cholangiocarcinoma}

Mass-forming CC are often seen in iCC and usually appear as $\mathrm{T} 1$ iso- or hypointense as well as T2 hyperintense [56]. Contrast enhancement of mass-forming iCC is variable. The most frequent pattern is peripheral enhancement on early images, which progresses on late images [57] (fig. 3). Contrast enhancement patterns depend on the tumour size, structure, and composition; small tumours with less fibrotic tissue may show homogeneous enhancement, whereas large fibrotic tumours may only enhance in late images.

When using hepatocyte-specific contrast agent, mass-forming iCC often has a cloud-like appearance with a central hyperintense area and a rim with lower signal intensity [58].

Extrahepatic ductal CC can also appear as a mass mimicking pancreatic adenocarcinoma. In these cases, assessment of the pancreatic duct on MRCP can help to differentiate both tumour entities.

Magnetic Resonance Imaging of Periductal Cholangiocarcinoma Periductal growth is usually seen in pCC and eCC [59]. Periductal CC grows alongside the wall of the bile duct, resulting in wall thickening and narrowing of the affected segment and dilation of the proximal intrahepatic bile ducts as seen on T2-weighted images and MRCP (fig. 4) [60]. Periductal CC usually shows slow contrast enhancement, which is best seen in late contrast-enhanced images [59].

\section{Magnetic Resonance Imaging of Intraductal \\ Cholangiocarcinoma}

Similar to mass-forming CC, intraductal CC begins to enhance on early post-contrast images, with peak enhancement on late post-contrast images [18]. MRCP is very suitable for the detection of intraductal CC, with a higher diagnostic accuracy when compared to CT $[18,48]$.

\section{Positron Emission Tomography/ Computed Tomography}

\section{Primary Staging}

$\mathrm{PET} / \mathrm{CT}$ is increasingly being recognized as a diagnostic tool in order to provide valuable information for the evaluation of CC. Regarding primary tumour detection in PET/CT, a meta-analysis of 1,232 patients in 23 studies provided high sensitivities of $95 \%$ and specificities of $83 \%$ for iCC [61], with a decrease to $84 \%$ and $76 \%$ in sensitivity and to $95 \%$ and $74 \%$ in specificity being found when evaluating pCC and eCC, respectively. This indicates a dependency of primary tumour evaluation on tumour localization. Other studies provide even lower sensitivities of 55\% for primary staging of pCC in ${ }^{18} \mathrm{~F}$-FDG ( ${ }^{18} \mathrm{~F}$-fluorodeoxyglucose) PET/CT [62]. Other false-negative issues include infiltrative growth, periductal sclerosing, and tumours with a high fraction of mucin [63]. The diagnostic performance of ${ }^{18} \mathrm{~F}-\mathrm{FDG} \mathrm{PET} / \mathrm{CT}$ in primary tumour evalua-
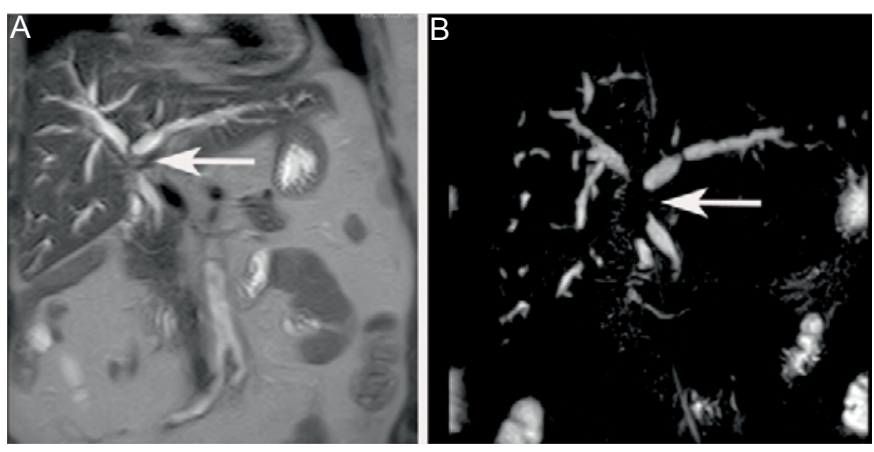

Fig. 4. A Coronal T2-weighted image and B MRCP of a 75-year-old female patient with periductal CC. The MRCP shows narrowing of the proximal biliary ducts (arrow) and dilation of the intrahepatic ducts.

tion of CC, however, depends on several aspects. First of all, there may be false-positive results due to concomitant inflammatory changes of the bile ducts, caused either by tumour-induced bile retention or chemotherapy or following invasive procedures such as implantation of intraluminal bile duct stents. Furthermore, ${ }^{18} \mathrm{~F}$ FDG PET/CT is limited in detecting small tumours, as only tumours $>1 \mathrm{~cm}$ in diameter are eligible for confident detection by this method $[64,65]$. Additionally, the different histopathological subtypes and the anatomical location of the tumour need to be considered when performing ${ }^{18} \mathrm{~F}$-FDG PET/CT.

Several studies indicate that in eCC especially, late ${ }^{18} \mathrm{~F}$-FDG PET imaging (image acquisition after injection $120 \mathrm{~min}$ and longer) could be helpful in differentiating benign bile duct strictures from malignant tumours. This theory is based on the continuous uptake of ${ }^{18} \mathrm{~F}$-FDG in malignant cells over $3 \mathrm{~h}$, whereas benign diseases tend to show a decline in ${ }^{18} \mathrm{~F}$-FDG uptake over time. This approach might be a helpful tool when using semiquantitative [66] or visual assessment [67], with a small increase in diagnostic sensitivity (76 vs. $79 \%$ ) and accuracy (76 vs. $80 \%$ ) being found, whereas specificity remained the same $(80 \%)$ in 39 patients, thereof 34 patients with CC [66]. Although some studies indicate a correlation between tumour-to-normal liver ratio (TNR) and standardized uptake values (SUV) in ${ }^{18} \mathrm{~F}-\mathrm{FDG}$ PET/CT (65 patients, thereof 47 CC patients) [68], other studies could not confirm this finding and did not find any correlation between tumour marker levels and SUV values (65 patients with ICC) [69]. However, it is worth noting that ${ }^{18} \mathrm{~F}$-FDG PET/CT-induced therapy regimens have been reported in $17-24 \%$ [69], indicating a substantial merit of this hybrid technique regarding the correct evaluation of $\mathrm{N}$ and $\mathrm{M}$ staging. Determination of $\mathrm{N}$ staging is crucial because it correlates with the 5-year survival rates which vary from $27 \%$ for N1 (regional lymph nodes in the hepatoduodenal ligament) to $50 \%$ for N0 [70]. Special emphasis lies on the detection of the N2 stage (lymph nodes periaortic, pericaval, or adjacent to the mesenteric or celiac artery), as these are being considered as a contraindication for tumour resection [70]. ${ }^{18} \mathrm{~F}-\mathrm{FDG}$ PET/CT showed a significantly higher specificity (88.2 vs. $64.7 \%$ ) and accuracy (75.9 vs. $60.9 \%$ ) for N staging in comparison to CT alone (sensitivity: PET/CT $80 \%$ vs. CT $20 \%$; specificity: 92.3 vs. $86.4 \%$, respectively) $[63,71]$. However, Kluge et 
Fig. 5. ${ }^{18} \mathrm{~F}-\mathrm{FDG}$-PET/CT for restaging of a 46-year-old male patient with iCC after chemotherapy. Hypodense iCC (A) with high tracer uptake indicating high metabolism of the lesion (B). After systemic therapy, central necrosis occurred (C) which cannot be visualized in CT alone (D).
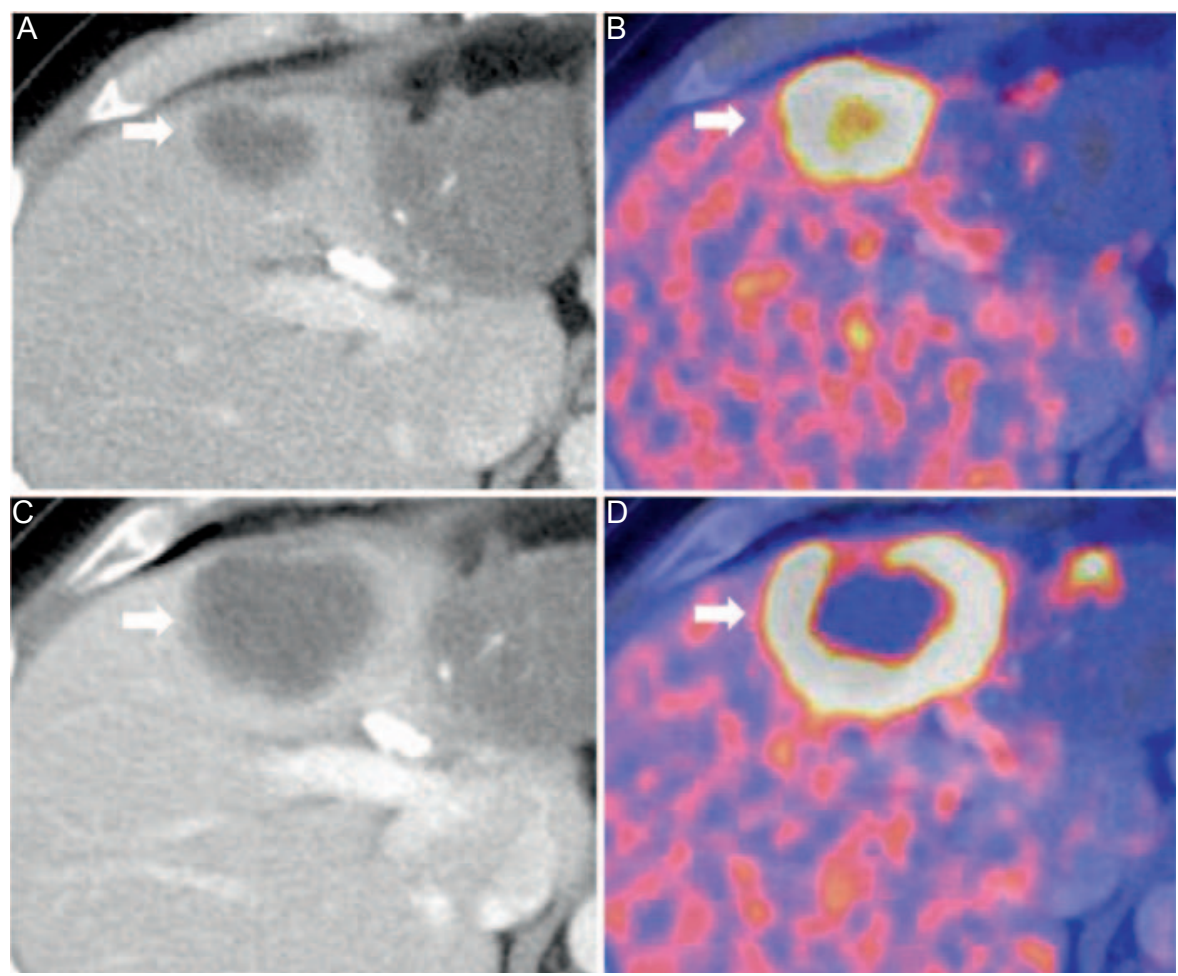

Fig. 6. 45-year-old male patient. Status post hemihepatectomy for pCC 3 years ago. In $\mathrm{CT}$, a small hypodense liver lesion adjacent to surgical clips is seen

(A) with elevated ${ }^{18} \mathrm{~F}$ FDG uptake (B) indicative for tumour recurrence. In addition to the local recurrence, two paracardial lymph nodes (C) show high FDG uptake in PET, which is suspicious for lymph node metastases (D).
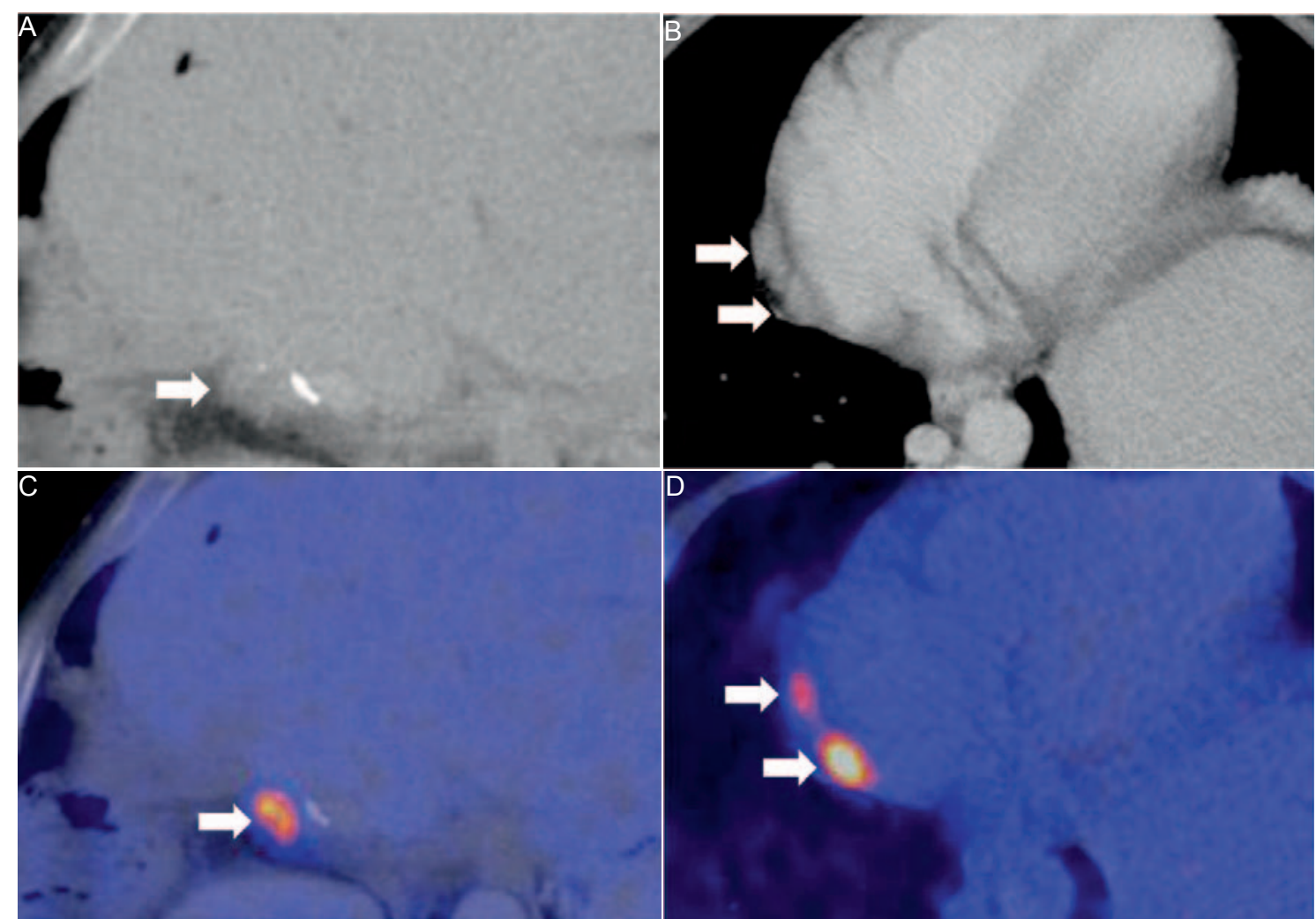

al. [72] examined the role of PET in the diagnosis and staging of $\mathrm{CC}$ and did not confirm a positive $\mathrm{N}$ staging in their collective of 26 patients; they did mention the significant influence of ${ }^{18} \mathrm{~F}-\mathrm{FDG}$ PET on M staging (metastases in liver, peritoneal cavity, distant lymph nodes, lung, bones, and brain), though. As advanced biliary cancer shows huge metabolic differences between the tumours [73] and detection of peritoneal carcinomatosis is difficult even in ${ }^{18} \mathrm{~F}$ FDG PET/CT imaging, the additional information of ${ }^{18} \mathrm{~F}-\mathrm{FDG}$ PET/CT regarding primary staging of CC in general was relatively low in comparison to conventional imaging including diagnostic 
Fig. 7. 49-year-old female patient after resection of pCC. In follow-up imaging, posttherapeutic changes were noticed at the resection margin (slight hyperintensity in the T2-weighted images of MRI; arrow). A Adjacent to surgical clips which are visible in $\mathrm{CT}$ (B). However, differentiation between local fibrotic changes and tumour recurrence was not possible based on the morphologic appearance in CT and MRI alone. In the fused ${ }^{18} \mathrm{~F}$-FDG-PET/CT, focal ${ }^{18} \mathrm{~F}$-FDG uptake was found in the corresponding area indicating tumour recurrence at the resection margin $(\mathbf{C})$.

laparoscopy [22]. However, the usefulness of preoperative ${ }^{18} \mathrm{~F}-\mathrm{FDG}$ $\mathrm{PET} / \mathrm{CT}$ in CC for $\mathrm{N}$ and $\mathrm{M}$ staging is stated in the National Comprehensive Cancer Network (NCCN) guidelines [74].

In conclusion, the evaluation of tumour resectability by means of different imaging methods reveals that CT imaging is the standard, with high reported sensitivities and specificities (for pCC: sensitivity $91-97 \%$, specificity $63-75 \%$ ) but with limitations in small hepatic lesions and small lymph node metastasis, where PET/CT seems a promising diagnostic tool. MRI provides comparable results (sensitivity $90-97 \%$, specificity $60-81 \%$ ), with limitations concerning the evaluation of the vessel involvement with a decreased sensitivity of $73 \%$ [75].

\section{Recurrent Disease}

Even in the $30 \%$ of patients with apparently primarily resectable tumours according to imaging, tumour recurrence occurs in up to $60-80 \%$ in the following 5 years [76]. The localization of tumour recurrence is mainly next to the resection zone and the hilum including locoregional lymph nodes [77]. Standardized imaging follow-up includes conventional imaging with CT or MRI [78]. In case of elevated tumour markers but negative conventional imaging, ${ }^{18} \mathrm{~F}$-FDG PET is helpful in the detection of recurrence (sensitivity $89 \%$, specificity $100 \%$; study with 33 patients) [79], with even higher values in ${ }^{18} \mathrm{~F}-\mathrm{FDG} \mathrm{PET} / \mathrm{CT}\left({ }^{18} \mathrm{~F}\right.$-FDG PET/CT sensitivity $94 \%$, specificity $100 \%$ vs. CT sensitivity $82 \%$, specificity $43 \%$; study with 16 patients). Seo et al. [65] suggested that PET/CT may also provide prognostic information as tumour recurrence could be predicted by ${ }^{18} \mathrm{~F}$-FDG PET/CT. This hypothesis is based on a study that found a lower disease-free survival if the primary tumour showed high metabolic activity with a cut-off value of $\mathrm{SUV}_{\text {mean }} 8.5$ (study based on 27 patients with iCC). Another study (25 patients) with distal bile duct adenocarcinoma revealed total lesion glycolysis (TLG) as a better prognostic marker in primarily resected patients than $\mathrm{SUV}_{\max }$ [80]. Onal et al. [81] reported that ${ }^{18} \mathrm{~F}-\mathrm{FDG}$ $\mathrm{PET} / \mathrm{CT}$ may be useful for radiation planning, with a possible dose reduction for adjacent organs at risk of $17 \%$ (right kidney, liver; study in 15 patients with eCC).

In conclusion, ${ }^{18} \mathrm{~F}$-FDG PET/CT seems to be a promising diagnostic tool in the primary staging of CC regarding $\mathrm{N}$ and $\mathrm{M}$ status. ${ }^{18} \mathrm{~F}-\mathrm{FDG}$ PET/CT plays a major role in the detection of tumour recurrence where this hybrid modality has great potential for differentiating between post-therapeutic changes (including fibroses due to radiotherapy) and tumour recurrence (fig. 5) [82]. Moreover, $\mathrm{PET} / \mathrm{CT}$ may be useful for the monitoring of treatment response, as metabolic changes may precede the anatomical ones $[83,84]$ (fig. 6, 7).

\section{Disclosure Statement}

The authors declare that no conflicts of interest exist.

\section{References}

1 Patel T, Singh P: Cholangiocarcinoma: emerging approaches to a challenging cancer. Curr Opin Gastroenterol 2007;23:317-323.

2 Shaib Y, El-Serag HB: The epidemiology of cholangiocarcinoma. Semin Liver Dis 2004;24:115-125.

3 Khan SA, Davidson BR, Goldin R, et al.; British Society of Gastroenterology: Guidelines for the diagnosis and treatment of cholangiocarcinoma: consensus document. Gut 2002;51(suppl 6):VI1-9.
4 Khan SA, Thomas HC, Davidson BR, Taylor-Robinson SD: Cholangiocarcinoma. Lancet 2005;366:1303-1314. 5 Shaib YH, Davila JA, McGlynn K, El-Serag HB: Rising incidence of intrahepatic cholangiocarcinoma in the United States: a true increase? J Hepatol 2004;40:472477.

6 Vitale A, Spolverato G, Bagante F, et al: A multi-institutional analysis of elderly patients undergoing a liver resection for intrahepatic cholangiocarcinoma. J Surg Oncol 2016;113:420-426.
7 Ghouri YA, Mian I, Blechacz B: Cancer review: Cholangiocarcinoma. J Carcinog 2015;14:1.

8 Plentz RR, Malek NP: Clinical presentation, risk factors and staging systems of cholangiocarcinoma. Best Pract Res Clin Gastroenterol 2015;29:245-252.

9 Yamasaki S: Intrahepatic cholangiocarcinoma: macroscopic type and stage classification. J Hepatobiliary Pancreat Surg 2003;10:288-291.

10 Lazaridis KN, Gores GJ: Cholangiocarcinoma. Gastroenterology 2005;128:1655-1667. 
11 Neitlich JD, Topazian M, Smith RC, et al: Detection of choledocholithiasis: comparison of unenhanced helical CT and endoscopic retrograde cholangiopancreatography. Radiology 1997;203:753-757.

12 Jung AY, Lee JM, Choi SH, et al: CT features of an intraductal polypoid mass: differentiation between hepatocellular carcinoma with bile duct tumor invasion and intraductal papillary cholangiocarcinoma. J Comput Assist Tomogr 2006;30:173-181.

13 Chung YE, Kim MJ, Park YN, et al: Varying appearances of cholangiocarcinoma: radiologic-pathologic correlation. Radiographics 2009;29:683-700.

14 Ayuso JR, Pages M, Darnell A: Imaging bile duct tumors: staging. Abdom Imaging 2013;38:1071-1081.

15 Bliznakova K, Kolev N, Buliev I, et al: Computer aided preoperative evaluation of the residual liver volume using computed tomography images. J Digit Imaging 2015;28:231-239.

16 Eshkenazy R, Dreznik Y, Lahat E, et al: Small for size liver remnant following resection: prevention and management. Hepatobiliary Surg Nutr 2014;3:303-312.

17 Zhang GQ, Zhang ZW, Lau WY, Chen XP: Associating liver partition and portal vein ligation for staged hepatectomy (ALPPS): a new strategy to increase resectability in liver surgery. Int J Surg 2014;12:437-441.

18 Sainani NI, Catalano OA, Holalkere NS, et al: Cholangiocarcinoma: current and novel imaging techniques. Radiographics 2008;28:1263-1287.

19 Shin DS, Ingraham CR, Dighe MK, et al: Surgical resection of a malignant liver lesion: what the surgeon wants the radiologist to know. AJR Am J Roentgenol 2014;203:W21-33.

20 Zhang Y, Uchida M, Abe T, et al: Intrahepatic peripheral cholangiocarcinoma: comparison of dynamic CT and dynamic MRI. J Comput Assist Tomogr 1999;23: 670-677.

21 Vilgrain V: Staging cholangiocarcinoma by imaging studies. HPB (Oxford) 2008;10:106-109.

22 Ruys AT, van Beem BE, Engelbrecht MR, et al: Radiological staging in patients with hilar cholangiocarcinoma: a systematic review and meta-analysis. Br J Radiol 2012;85:1255-1262.

23 Cho ES, Park MS, Yu JS, et al: Biliary ductal involvement of hilar cholangiocarcinoma: multidetector computed tomography versus magnetic resonance cholangiography. J Comput Assist Tomogr 2007;31:72-78.

24 Kim HM, Park JY, Kim KS, et al: Intraductal ultrasonography combined with percutaneous transhepatic cholangioscopy for the preoperative evaluation of longitudinal tumor extent in hilar cholangiocarcinoma. J Gastroenterol Hepatol 2010;25:286-292.

25 Bismuth H, Nakache R, Diamond T: Management strategies in resection for hilar cholangiocarcinoma. Ann Surg 1992;215:31-38.

26 Edge SB, Compton CC: The American Joint Committee on Cancer: the 7th edition of the AJCC cancer staging manual and the future of TNM. Ann Surg Oncol 2010;17:1471-1474.

27 Chung YE, Kim MJ, Park YN, et al: Staging of extrahepatic cholangiocarcinoma. Eur Radiol 2008;18:21822195.

28 Uchida M, Ishibashi M, Tomita N, et al: Hilar and suprapancreatic cholangiocarcinoma: value of $3 \mathrm{D}$ angiography and multiphase fusion images using MDCT. AJR Am J Roentgenol 2005;184:1572-1577.

29 Asayama Y, Yoshimitsu K, Irie H, et al: Delayed-phase dynamic CT enhancement as a prognostic factor for mass-forming intrahepatic cholangiocarcinoma. Radiology 2006;238:150-155.

30 Kim SA, Lee JM, Lee KB, et al: Intrahepatic massforming cholangiocarcinomas: enhancement patterns at multiphasic CT, with special emphasis on arterial enhancement pattern - correlation with clinicopathologic findings. Radiology 2011;260:148-157.
31 Nanashima A, Abo T, Murakami G, et al: Intrahepatic cholangiocarcinoma: relationship between tumor imaging enhancement by measuring attenuation and clinicopathologic characteristics. Abdom Imaging 2013; 38:785-792.

32 Blechacz BR, Gores GJ: Cholangiocarcinoma. Clin Liver Dis 2008; 12:131-150, ix

33 Lee HY, Kim SH, Lee JM, et al: Preoperative assessment of resectability of hepatic hilar cholangiocarcinoma: combined CT and cholangiography with revised criteria. Radiology 2006;239:113-121.

34 Hyder O, Hatzaras I, Sotiropoulos GC, et al: Recurrence after operative management of intrahepatic cholangiocarcinoma. Surgery 2013;153:811-818.

35 Baheti AD, Tirumani SH, Rosenthal MH, et al: Diagnosis and management of intrahepatic cholangiocarcinoma: a comprehensive update for the radiologist. Clin Radiol 2014;69:e463-470.

36 Kim JE, Lee JM, Baek JH, et al: Initial assessment of dual-energy CT in patients with gallstones or bile duct stones: can virtual nonenhanced images replace true nonenhanced images? AJR Am J Roentgenol 2012;198: $817-824$.

37 Schabel C, Bongers M, Sedlmair M, et al: Assessment of the hepatic veins in poor contrast conditions using dual energy CT: evaluation of a novel monoenergetic extrapolation software algorithm. Rofo 2014;186:591597.

38 Bongers MN, Schabel C, Krauss B, et al: Noise-optimized virtual monoenergetic images and iodine maps for the detection of venous thrombosis in second-generation dual-energy CT (DECT): an ex vivo phantom study. Eur Radiol 2015;25:1655-1664.

39 Marin D, Ramirez-Giraldo JC, Gupta S, et al: Effect of a noise-optimized second-generation monoenergetic algorithm on image noise and conspicuity of hypervascular liver tumors: an in vitro and in vivo study. AJR Am J Roentgenol 2016;206:1222-1232.

40 Wang Q, Shi G, Qi X, et al: Quantitative analysis of the dual-energy CT virtual spectral curve for focal liver lesions characterization. Eur J Radiol 2014;83:1759-1764.

41 Fischer MA, Reiner CS, Raptis D, et al: Quantification of liver iron content with CT-added value of dual-energy. Eur Radiol 2011;21:1727-1732.

42 Fischer MA, Gnannt R, Raptis D, et al: Quantification of liver fat in the presence of iron and iodine: an exvivo dual-energy CT study. Invest Radiol 2011;46: 351-358.

43 Oğul H, Kantarcı M, Genç B, et al: Perfusion CT imaging of the liver: review of clinical applications. Diagn Interv Radiol 2014;20:379-389.

44 Miles KA, Hayball MP, Dixon AK: Functional images of hepatic perfusion obtained with dynamic CT. Radiology 1993;188:405-411.

45 Bongers MN, Bier G, Marcus R, et al: Image quality of a novel frequency selective nonlinear blending algorithm: an ex vivo phantom study in comparison to single-energy acquisitions and dual-energy acquisitions with monoenergetic reconstructions. Invest Radiol 2016;51:647-654.

46 Low RN: Abdominal MRI advances in the detection of liver tumours and characterisation. Lancet Oncol 2007; 8:525-535.

47 Khan SA, Davidson BR, Goldin RD, et al.; British Society of Gastroenterology: Guidelines for the diagnosis and treatment of cholangiocarcinoma: an update. Gut 2012;61:1657-1669.

48 Jhaveri KS, Hosseini-Nik H: MRI of cholangiocarcinoma. J Magn Reson Imaging 2015;42:1165-1179.

49 Goodwin MD, Dobson JE, Sirlin CB, et al: Diagnostic challenges and pitfalls in MR imaging with hepatocytespecific contrast agents. Radiographics 2011;31:15471568.
50 Irie $\mathrm{H}$, Honda $\mathrm{H}$, Tajima T, et al: Optimal MR cholangiopancreatographic sequence and its clinical application. Radiology 1998;206:379-387.

51 Romagnuolo J, Bardou M, Rahme E, et al: Magnetic resonance cholangiopancreatography: a meta-analysis of test performance in suspected biliary disease. Ann Intern Med 2003;139:547-557.

52 Cui XY, Chen HW: Role of diffusion-weighted magnetic resonance imaging in the diagnosis of extrahepatic cholangiocarcinoma. World J Gastroenterol 2010;16:3196-3201.

53 Park HS, Lee JM, Choi JY, et al: Preoperative evaluation of bile duct cancer: MRI combined with MR cholangiopancreatography versus MDCT with direct cholangiography. AJR Am J Roentgenol 2008;190:396-405.

54 Sahani D, Mehta A, Blake M, et al: Preoperative hepatic vascular evaluation with CT and MR angiography: implications for surgery. Radiographics 2004;24: 1367-1380.

55 Razumilava N, Gores GJ, Lindor KD: Cancer surveillance in patients with primary sclerosing cholangitis. Hepatology 2011;54:1842-1852.

56 Vilgrain V, Van Beers BE, Flejou JF, et al: Intrahepatic cholangiocarcinoma: MRI and pathologic correlation in 14 patients. J Comput Assist Tomogr 1997;21:59-65.

57 Maetani Y, Itoh K, Watanabe C, et al: MR imaging of intrahepatic cholangiocarcinoma with pathologic correlation. AJR Am J Roentgenol 2001;176:1499-1507.

$58 \mathrm{Kim} \mathrm{SH}$, Lee $\mathrm{CH}$, Kim BH, et al: Typical and atypical imaging findings of intrahepatic cholangiocarcinoma using gadolinium ethoxybenzyl diethylenetriamine pentaacetic acid-enhanced magnetic resonance imaging. J Comput Assist Tomogr 2012;36:704-709.

59 Vanderveen KA, Hussain HK: Magnetic resonance imaging of cholangiocarcinoma. Cancer Imaging 2004; 104-115.

60 Weinbren K, Mutum SS: Pathological aspects of cholangiocarcinoma. J Pathol 1983;139:217-238.

61 Annunziata S, Caldarella C, Pizzuto DA, et al: Diagnostic accuracy of fluorine-18-fluorodeoxyglucose positron emission tomography in the evaluation of the primary tumor in patients with cholangiocarcinoma: a meta-analysis. Biomed Res Int 2014;2014:247693.

62 Ringe KI, Wacker F: Radiological diagnosis in cholangiocarcinoma: application of computed tomography, magnetic resonance imaging, and positron emission tomography. Best Pract Res Clin Gastroenterol 2015; 29:253-265.

63 Kim JY, Kim MH, Lee TY, et al: Clinical role of ${ }^{18} \mathrm{~F}$ FDG PET-CT in suspected and potentially operable cholangiocarcinoma: a prospective study compared with conventional imaging. Am J Gastroenterol 2008; 103:1145-1151.

64 Anderson CD, Rice MH, Pinson CW, et al: Fluorodeoxyglucose PET imaging in the evaluation of gallbladder carcinoma and cholangiocarcinoma. J Gastrointest Surg 2004;8:90-97.

65 Seo S, Hatano E, Higashi T, et al: Fluorine-18 fluorodeoxyglucose positron emission tomography predicts lymph node metastasis, P-glycoprotein expression, and recurrence after resection in mass-forming intrahepatic cholangiocarcinoma. Surgery 2008;143:769-777.

66 Choi EK, Yoo IeR, Kim SH, et al: The clinical value of dual-time point ${ }^{18} \mathrm{~F}$-FDG PET/CT for differentiating extrahepatic cholangiocarcinoma from benign disease. Clin Nucl Med 2013;38:e106-111.

67 Nishiyama Y, Yamamoto Y, Kimura N, et al: Comparison of early and delayed FDG PET for evaluation of biliary stricture. Nucl Med Commun 2007;28:914-919.

68 Alkhawaldeh K, Faltten S, Biersack HJ, Ezziddin S: The value of F-18 FDG PET in patients with primary sclerosing cholangitis and cholangiocarcinoma using visual and semiquantitative analysis. Clin Nucl Med 2011; 36:879-883. 
69 Jiang L, Tan H, Panje CM, et al: Role of ${ }^{18} \mathrm{~F}$-FDG PET/ CT imaging in intrahepatic cholangiocarcinoma. Clin Nucl Med 2016;41:1-7.

70 Mar WA, Shon AM, Lu Y, et al: Imaging spectrum of cholangiocarcinoma: role in diagnosis, staging, and posttreatment evaluation. Abdom Radiol (NY) 2016 41:553-567.

71 Park TG, Yu YD, Park BJ, et al: Implication of lymph node metastasis detected on ${ }^{18} \mathrm{~F}$-FDG PET/CT for surgical planning in patients with peripheral intrahepatic cholangiocarcinoma. Clin Nucl Med 2014;39:1-7.

72 Kluge R, Schmidt F, Caca K, et al: Positron emission tomography with $\left[{ }^{18} \mathrm{~F}\right]$ fluoro-2-deoxy-D-glucose for diagnosis and staging of bile duct cancer. Hepatology 2001;33:1029-1035.

73 Cho KM, Oh DY, Kim TY, et al: Metabolic characteristics of advanced biliary tract cancer using ${ }^{18} \mathrm{~F}$-fluorodeoxyglucose positron emission tomography and their clinical implications. Oncologist 2015;20:926-933.

74 National Comprehensive Cancer Network. Hepatobiliary Cancers. Version 1.2016. www.nccn.org/professionals/physician_gls/PDF/hepatobiliary.pdf.
5 Zhang H, Zhu J, Ke F, et al: Radiological imaging for assessing the respectability of hilar cholangiocarcinoma: a systematic review and meta-analysis. Biomed Res Int 2015;2015:497942.

76 Brandi G, Venturi M, Pantaleo MA, et al: Cholangiocarcinoma: current opinion on clinical practice diagnostic and therapeutic algorithms: a review of the literature and a long-standing experience of a referral center. Dig Liver Dis 2016;48:231-241.

77 Yamamoto M, Takasaki K, Otsubo T, et al: Recurrence after surgical resection of intrahepatic cholangiocarcinoma. J Hepatobiliary Pancreat Surg 2001;8:154-157.

78 Jung SJ, Woo SM, Park HK, et al: Patterns of initial disease recurrence after resection of biliary tract cancer. Oncology 2012;83:83-90.

79 Corvera CU, Blumgart LH, Akhurst T, et al: ${ }^{18} \mathrm{~F}-$ fluorodeoxyglucose positron emission tomography influences management decisions in patients with biliary cancer. J Am Coll Surg 2008;206:57-65.

80 Lee EJ, Chang SH, Lee TY, et al: Prognostic value of FDG-PET/CT total lesion glycolysis for patients with resectable distal bile duct adenocarcinoma. Anticancer Res 2015;35:6985-6991.
81 Onal C, Topuk S, Yapar AF, et al: Comparison of computed tomography- and positron emission tomography-based radiotherapy planning in cholangiocarcinoma. Onkologie 2013;36:484-490.

82 Cameron K, Golan S, Simpson W, et al: Recurrent pancreatic carcinoma and cholangiocarcinoma: ${ }^{18} \mathrm{~F}$ fluorodeoxyglucose positron emission tomography/ computed tomography (PET/CT). Abdom Imaging 2011;36:463-471.

83 Jadvar H, Henderson RW, Conti PS: [F-18]fluorodeoxyglucose positron emission tomography and positron emission tomography: computed tomography in recurrent and metastatic cholangiocarcinoma. J Comput Assist Tomogr 2007;31:223-228.

84 Donswijk ML, Hess S, Mulders T, Lam MG: $\left[{ }^{18} \mathrm{~F}\right]$ Fluorodeoxyglucose PET/computed tomography in gastrointestinal malignancies. PET Clin 2014;9:421441, v-vi. 